\title{
An image is worth a thousand words of public health
}

\author{
Uma imagem vale por mil palavras em saúde pública
}

Janet Treasure?

Eating disorders, anorexia nervosa and bulimia nervosa are common disorders found in nearly $10 \%$ of young women. There is a large range in clinical severity. Some cases are mild and transient. However for others the quality of life for the individual and her family shrinks. The physical and psychosocial morbidity is high. Family members also have clinical levels of anxiety. The costs for the individual, the family and society are huge.

Anorexia nervosa has a history stretching back over the centuries. In contrast, all epidemiological studies have shown that the incidence of the binge eating disorders (including bulimia nervosa) has rapidly increased in the last half of the twentieth century (Hudson et al., 2007). Most experts agree that cultural factors in terms of eating behaviours and values about weight and shape are important causal and maintaining elements in the bingeing disorders. The internalisation of the thin ideal and the stigmatisation of overweight with negative comments about shape and weight are core risk factors (Taylor et al., 2006a). Dieting to attain this idealized form can trigger an erratic pattern of eating especially if it is used in combination with extreme behaviours that compensate for overeating.

Studies in animals suggest that persistent changes in the brain and behaviour, like those seen in the addictions, result if the pattern of eating is disrupted. Thus rats can be exposed to environmental conditions that cause them not only to binge eat but also to be more susceptible to addiction to amphetamine (Corwin, 2006). The environmental setting conditions include: semi-starvation with intermittent access to high palatability food (cookies), having their stomach contents drained out, and exposure to stress. A young woman harassed by explicit and implicit messages of body hatred may easily fall into this behavioural trap. This will cause her incentive system to be permanently imprinted by this experience, especially if it occurs during the critical period of adolescent brain development, leaving her appetite and emotional systems dysregulated. The paradox can be that a desire to be thin can set in train a pattern of disturbed eating which increases the risk for obesity. Society needs to think how it can protect young people from these consequences.

Excellent prevention work is taking place in schools (Neumark-Sztainer, 2005) and colleges. For example, an educational web-based intervention promoting a healthy relationship with food and body image can prevent the onset of an eating disorder in those that are at highest risk (Taylor et al., 2006b). However the case for public health interventions seems clear and there are several key targets. Women born from 1950 onwards have been exposed to a culture of conflicting forces. Never before has so much high palatability food been so readily accessible. The social rules of eating, such as breakfast, and a joint communion around meals, have been broken down. We are encouraged to eat on the move or isolated at our computer desks or armchairs in front of the TV. The gradual increase in body mass index (BMI) in the populations caused by these changes is pitted against fashionable images. Capitalism flourishes on making us desire what we do not currently have. Hence the current allure of emaciation. Marketing and advertisements distort the human body with products such as Photoshop and airbrushing to produce unrealistic images that tap into primitive 
emotions. A large head on a small body is the classical form of a baby or young animal and has innate appeal. The market for diet products and plastic surgery flourishes.

Spain has taken the first step. The Health Authorities of the Region of Madrid and the Annual Cibeles Fashion Show (Pasarela Cibeles) banned extremely thin models from participating in this year's event. Models with a BMI below $18 \mathrm{~kg} / \mathrm{m}^{2}$ (30\% of the participants) were offered medical help rather than a position on the cat walk. Other countries such as the UK shy away from direct intervention. However health and safety legislation possibly could be used to protect the young women in this industry from being encouraged to adopt unhealthy behaviours to attain unrealistic shapes. The "image" industry should be discouraged, however and whenever possible, from making icons out of emaciated models.

Some people dismiss the interest in size zero models as irrelevant whimsy. After all, human beings over time have done, or had done, extraordinary things to their body for cultural reasons. However never was there a time when images have such a global reach. Moreover the consequences are more than skin deep. Tampering with appetite regulation at the critical period of development can damage health in the long term. If there is a will, changes can be made for the public good; there was a time when smoking looked cool too.

\section{REFERENCES}

Corwin RL. Bingeing rats: a model of intermittent excessive behavior? Appetite, 46: 11-5, 2006.

Hudson JI, Hiripi E, Pope HG Jr, Kessler RC. The prevalence and correlates of eating disorders in the national comorbidity survey replication. Biol Psychiatry, 61: 348-58, 2007.

Neumark-Sztainer D. Preventing the broad spectrum of weight-related problems: working with parents to help teens achieve a healthy weight and a positive body image. J Nutr Educ Behav 37 Suppl 2: S133-S140, 2005.

Taylor CB, Bryson S, Celio Doyle AA, Luce KH, Cunning D, Abascal LB et al. The adverse effect of negative comments about weight and shape from family and siblings on women at high risk for eating disorders. Pediatrics, 118: 731-8, 2006a.

Taylor CB, Bryson S, Luce KH, Cunning D, Doyle AC, Abascal LB et al. Prevention of eating disorders in at-risk college-age women. Arch Gen Psychiatry, 63: 881-8, 2006b. 